\title{
Analytic Design Procedure of Three-mirror Telescope Corrected for Spherical Aberration, Coma, Astigmatism, and Petzval Field Curvature
}

\author{
Jong Ung Lee* \\ Department of Laser and Optical Information Engineering, Cheongju University, Cheongju 360-764, Korea \\ Seung Moon Yu \\ Advanced $R$ \& $D$ Team, Korea Electro Optic. Co. Ltd, Buchoen 421-809, Korea
}

(Received May 6, 2009 : accepted May 20, 2009)

\begin{abstract}
There are total eight degrees of freedom in designing a three-mirror system. If we correct four kinds of third order aberrations and the system should have the specified effective focal length, the remaining three degrees of freedom can be used for selecting a suitable configuration for a specific application. We suggest an analytic design procedure for a three-mirror telescope system which has a suitably sized secondary mirror and proper separations between mirrors, and is corrected for four kinds of third order aberrations, spherical aberration, coma, astigmatism, and field curvature. Two design examples are shown. One has a compact configuration with off-axial field, the other has relatively long configuration with annular ring field.
\end{abstract}

Keywords: Three-mirror system, Telescope, Optical system design

OCIS codes : (110.6770) Telescopes; (120.4570) Optical design of instruments; (220.2740) Geometrical optics, optical design; (220.4830) Optical systems design; (350.6090) Space optics

\section{INTRODUCTION}

Reflecting optical systems with conic mirrors are widely used for large astronomical telescopes and satellite cameras because the conic surface can be fabricated very precisely[1]. For space optics, single mirror systems and two mirror systems were used for small field or low resolution imaging [2,3]. Recent development in sensor technology permits us wider field and higher resolution in optical observation. Therefore, three mirror systems are becoming popular for satellite camera systems.

Analytic methods to correct the third order aberrations of a three-mirror system have been reported by D. Korsh in 1972[4] and P. N. Robb in 1978[5]. In D. Korsh's paper, the conditions for correcting third order spherical aberration, coma, astigmatism and Petzval field curvature are expressed as functions of $m_{i}$ and $p_{i}$, where $m_{i}$ is the transverse magnification of the i-th surface about the

*Corresponding author: julee@cju.ac.kr marginal ray, and $p_{i}$ is the transverse magnification of i-th surface about the principal ray. The marginal ray has information about object imaging, and the principal ray has information about pupil imaging. These formulae are very useful to correct aberrations, but we can not know shapes and locations of the mirrors because the $\left(m_{i}, p_{i}\right)$ are not familiar design variables such as curvature radius $r_{i}$ and separation $d_{i}$. In P. N. Robb's paper [5], the third order aberrations are expressed as functions of curvature $c_{i}$ and axial distance $d_{i}$, so we can interpret the configuration of the system more easily. However, the procedures for aberration correction are based on paraxial ray tracing. We can get only a numerical solution. More simple analytic expressions for the third order aberrations of three-mirror systems were presented in 1995[6,7]. The aberrations are expressed as functions of $\left(m_{i}, a_{i}\right)$. The $a_{i}$ is defined as the ratio $h_{i+1} / h_{i}$, where $h_{i}, h_{i+1}$ are the incident heights of the marginal ray on the i-th surface and the following surface respectively. 
In designing a three-mirror system, we have eight degrees of freedom, three curvature radii $\left(r_{1}, r_{2}, r_{3}\right)$, three conic constants $\left(k_{1}, k_{2}, k_{3}\right)$ and two axial separations between mirrors $\left(d_{1}, d_{2}\right)$. Among the five kinds of third order aberrations, spherical aberration, coma, astigmatism and distortion are linear functions of the conic constants $[7,8]$. Three kinds of the third order aberrations can be corrected by using the conic constants. We need two degrees of freedom to satisfy the focal length requirement and to correct Petzval field curvature. Hence, the residual three degrees of freedom can be used for selecting a suitable configuration.

We present an analytic design procedure for a threemirror system which has a suitable configuration for real applications and is corrected for four of the third order aberrations, spherical aberration, coma, astigmatism and Petzval field curvature. Three design parameters $\left(h_{2}, d_{1}, d_{2}\right)$ are selected to get a suitable configuration, where $h_{2}$ is the incident height of the marginal ray on the secondary mirror. The $\left(h_{2}, d_{1}\right)$ determine the primary mirror and central obstruction by the secondary mirror, and $d_{2}$ is directly related to instrumental space. The condition for correcting Petzval field curvature is given by a quadratic equation. Two analytic solutions may be possible. They have the same primary mirror, central obstruction, and axial separations. But, they have different curvature radii $\left(r_{2}, r_{3}\right)$, back focal length(BFL), exit pupil position and intermediate image plane of the secondary mirror. These are very important for constructing a real telescope system. Since the focal point $F^{\prime}$ is located in front of the tertiary mirror, we need at least a folding mirror to access the focus. There are two possible positions to locate the folding mirror. One position is the intermediate image plane of the secondary mirror, the other is the exit pupil. The former configuration has the advantage of a compact design compared with the other case, but its disadvantage is the relatively narrow field. Flat field anastigmat in Ref. [8] and HiRISE in Ref. [9] are adopting this type of folding mirror. The latter configuration needs relatively large instrumental space, but this system allows more wide field. Ref. [10] and Ref. [11] are the examples of this configuration. To get a practical design that we want, a proper combination of $\left(h_{2}, d_{1}, d_{2}\right)$ should be selected. As design examples, both of the configurations are presented. We call the system for which the folding mirror is located at the intermediate image plane of the secondary mirror "object conjugation type", because the intermediate image plane is the conjugate plane of object and the folding mirror acts as a field stop. The system for which the folding mirror is located at the exit pupil will be called "pupil conjugation type". The position is the conjugate plane of the entrance pupil, and the folding mirror acts as a glare stop. This is another benefit of the pupil conjugation type.

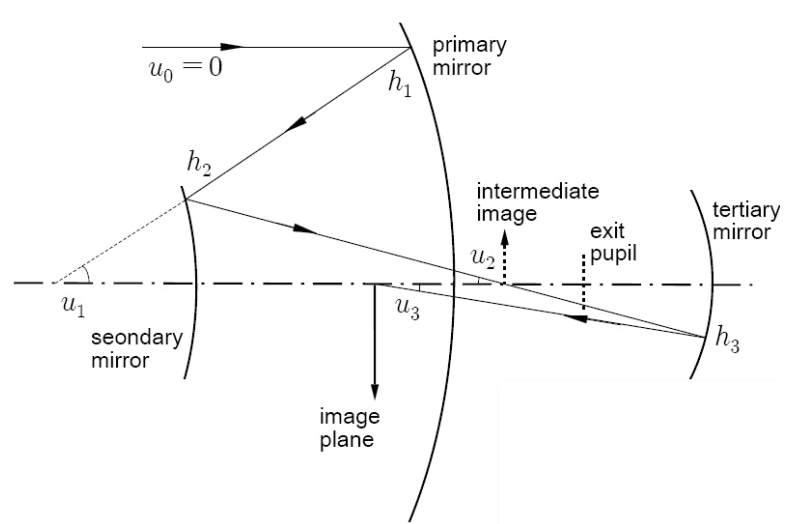

(a) marginal ray

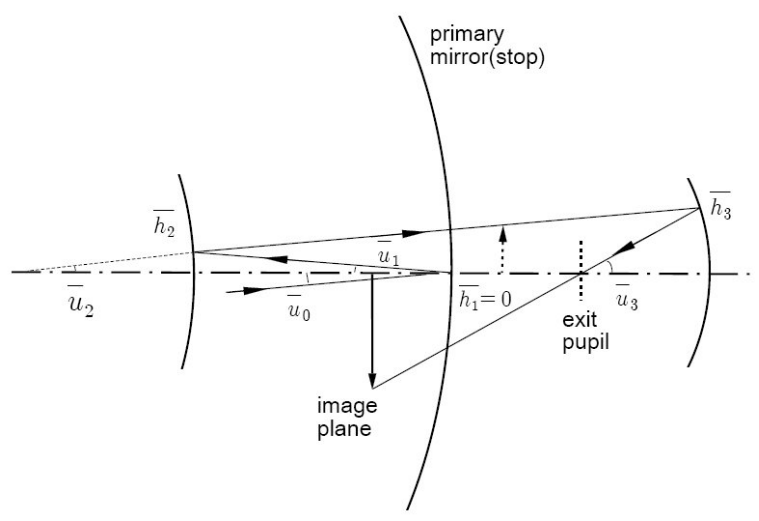

(b) principal ray

FIG. 1. Optical layout and rays of three-mirror system.

\section{DESIGN PROCEDURE OF THREE-MIRROR SYSTEM}

\section{First Order Design}

Figure 1 shows a marginal ray and a chief ray of a three-mirror system. The primary mirror is the stop of this system. In Figure 1(a), $\left(h_{1}, h_{2}, h_{3}\right)$ are incident heights of the marginal ray, and $\left(u_{0}, u_{1}, u_{2}, u_{3}\right)$ are paraxial angles of the ray after reflection. There are two kinds of sign convention for the paraxial angle $u_{i}$. We are adopting the sign convention of $\mathrm{W}$. T. Welford [12]. For the cases in Figure 1(a), $u_{1}$ has positive sign, $u_{2}$ and $u_{3}$ have negative signs. In Figure $1(\mathrm{~b}), \bar{h}_{i}$ and $\bar{u}_{i}$ are the incident height and paraxial angle of the chief ray respectively. If the incident heights $\left(h_{1}, h_{2}, h_{3}\right)$ and the incident angles $\left(u_{0}, u_{1}, u_{2}, u_{3}\right)$ are given, the curvature radius of i-th mirror $r_{i}$ and axial distance $d_{i}$ are obtained as follows:

$$
\begin{aligned}
& \frac{1}{r_{i}}=-\frac{u_{i}+u_{i-1}}{2 h_{i}} \\
& d_{i}=\frac{h_{i+1}-h_{i}}{u_{i}}
\end{aligned}
$$


Effective focal length $f^{\prime}$, f-number $F_{N}$, and half field angle $\beta$ are always given in the specifications for design. Let's assume that the object plane is located at infinity and the stop is located on the primary mirror. Some of the ray parameters can be determined easily from the specifications.

$$
\begin{aligned}
& h_{1}=\frac{\left|f^{\prime}\right|}{2 F_{N}} \\
& u_{0}=0 \\
& u_{3}= \pm \frac{2}{F_{N}} \\
& \bar{u}_{0}=-\bar{u}_{1}=\tan \beta
\end{aligned}
$$

In this study, $\left(h_{2}, d_{1}, d_{2}\right)$ are taken as basic design parameters to get a suitable configuration. Clear aperture of the secondary mirror $\Phi_{2}$ can be evaluated approximately as

$$
\Phi_{2}=2\left(\left|h_{2}\right|+\left|\bar{h}_{2}\right|\right)
$$

where $\overline{h_{2}}$ is the incident height of the chief ray on the secondary mirror. It is given by

$$
\bar{h}_{2}=d_{1} \bar{u}_{1}=-d_{1} \tan \beta
$$

The central obstruction by the secondary mirror $\sigma$ can be expressed by

$$
\sigma=\frac{\Phi_{2}}{2 h_{1}}=\left|\frac{h_{2}}{h_{1}}\right|+\left|\frac{d_{1} \tan \beta}{h_{1}}\right|
$$

From Eq. (1) and Eq. (2), f-ratio of the primary mirror $F_{1}$ and the curvature radius of the primary mirror $r_{1}$ are also determined as follows:

$$
\begin{aligned}
& u_{1}=-\frac{h_{2}-h_{1}}{d_{1}} \\
& F_{1}=\left|\frac{1}{2 u_{1}}\right| \\
& \frac{1}{r_{1}}=\frac{u_{1}}{2 h_{1}}
\end{aligned}
$$

Among the marginal ray parameters, $\left(h_{3}, u_{2}\right)$ are not determined yet. If we determine $u_{2}$ by using the condition for correcting Petzval field curvature, $h_{3}, r_{2}$ and $r_{3}$ will be given by

$$
\begin{aligned}
& h_{3}=h_{2}+d_{2} u_{2} \\
& \frac{1}{r_{2}}=-\frac{u_{2}+u_{1}}{2 h_{2}} \\
& \frac{1}{r_{3}}=-\frac{u_{3}+u_{2}}{2 h_{3}}
\end{aligned}
$$

\section{Correction of Petzval Field Curvature}

Petzval field curvature $S_{I V}$ is given by [12]

$$
S_{I V}=-H^{2} \sum \frac{1}{r_{i}}\left(\frac{1}{n_{i}}-\frac{1}{n_{i-1}}\right)
$$

where $H$ is the Lagrange's invariant of the system, and $n_{i}$ is the refractive index after refraction. In a threemirror system, the condition for correcting Petzval field curvature can be expressed as follows

$$
\frac{1}{r_{1}}-\frac{1}{r_{2}}+\frac{1}{r_{3}}=\frac{u_{1}+u_{0}}{2 h_{1}}-\frac{u_{2}+u_{1}}{2 h_{2}}+\frac{u_{2}+u_{3}}{2 h_{3}}=0
$$

There are two undetermined parameters $u_{2}, h_{3}$ in Eq. (15), but $h_{3}$ can be expressed as a function of $u_{2}$ by using Eq. (11). Hence, Eq. (15) can be rewritten as a quadratic equation of $u_{2}$.

$$
h_{1} d_{2} u_{2}^{2}-d_{1} d_{2} u_{1}^{2} u_{2}-h_{2}\left(d_{1} u_{1}^{2}+h_{1} u_{3}\right)=0
$$

To get real solutions, the discriminant of Eq. (16) $D$ must be zero or positive.

$$
D=d_{1}^{2} d_{2}^{2} u_{1}^{4}+4 h_{1} h_{2} d_{2}\left(d_{1} u_{1}^{2}+h_{1} u_{3}\right) \geq 0
$$

There are two possible solutions, let's denote them the solution $u_{2+}$ and $u_{2}$.

$$
\begin{aligned}
& u_{2+}=\frac{d_{1} d_{2} u_{1}^{2}+\sqrt{D}}{2 h_{1} d_{2}} \\
& u_{2-}=\frac{d_{1} d_{2} u_{1}^{2}-\sqrt{D}}{2 h_{1} d_{2}}
\end{aligned}
$$

The two solutions have the same primary mirror, central obstruction, and axial separations since these are specified by the design parameters $\left(h_{2}, d_{1}, d_{2}\right)$. But, the curvature radii $\left(r_{2}, r_{3}\right)$, the back focal length(BFL), position of the exit pupil and intermediate image plane of the secondary mirror are different. They depend on the solution of $u_{2}$.

\section{Correction of Spherical Aberration, Coma and Astigmatism}


The third order spherical aberration $S_{I}$, coma $S_{I I}$, and astigmatism $S_{I I I}$ of a three-mirror system are linear functions of the conic constants $k_{1}, k_{2}$ and $k_{3}$. They can be expressed as follows $[6,7,8]$ :

$$
\begin{aligned}
S_{I}= & -\frac{1}{4} h_{1} u_{3}^{3}\left\{-m_{3}^{3} s_{1 f}+a_{1} a_{2} s_{1 r}-m_{2}^{3} m_{3}^{3} k_{1}+\right. \\
& \left.a_{1}\left(m_{2}-1\right)^{3} m_{3}^{3} k_{2}+a_{1} a_{2}\left(m_{3}-1\right)^{3} k_{3}\right\} \\
S_{I I}= & -\frac{1}{4} H u_{3}^{2}\left\{m_{3}^{2} s_{2 f}+s_{2 r}+Q s_{1 r}+\right. \\
S_{I I}= & -\frac{1}{4} H u_{3}^{2}\left\{m_{3}^{2} s_{2 f}+s_{2 r}+Q s_{1 r}+\right. \\
+ & \left.\frac{\left(a_{1}-1\right)\left(m_{2}-1\right)^{3} m_{3}^{2}}{m_{2}} k_{2}+Q\left(m_{3}-1\right)^{3} k_{3}\right\} \\
S_{I I I}= & -\frac{H^{2} u_{3}}{4 h_{1}}\left\{-m_{3} s_{3 f}+\frac{s_{3 r}+2 Q s_{2 r}+Q^{2} s_{i r}}{a_{1} a_{2}}+\right. \\
& \left.\frac{\left(a_{1}-1\right)^{2}\left(m_{2}-1\right)^{3} m_{3}}{a_{1} m_{2}^{2}} \kappa_{2}+\frac{Q^{2}\left(m_{3}-1\right)^{3}}{a_{1} a_{2}} \kappa_{3}\right\}
\end{aligned}
$$

where,

$$
\begin{aligned}
& s_{1 f}=m_{2}^{3}+a_{1}\left(1+m_{2}\right)^{2}\left(1-m_{2}\right) \\
& s_{1 r}=-\left(1+m_{3}\right)^{2}\left(1-m_{3}\right) \\
& s_{2 f}=-2 m_{2}^{2}-\frac{\left(1-m_{2}^{2}\right)\left\{m_{2}-1+\left(1+m_{2}\right) a_{1}\right\}}{m_{2}} \\
& s_{2 r}=-2\left(1-m_{3}^{2}\right) \\
& Q=\frac{a_{2}\left(a_{1}-1+m_{2}\right)-m_{2}}{m_{2} m_{3}} \\
& s_{3 f}=4 m_{2}+\frac{\left(1-m_{2}\right)\left\{m_{2}-1+\left(1+m_{2}\right) a_{1}\right\}^{2}}{m_{2}^{2} a_{1}} \\
& s_{3 r}=-4\left(1-m_{3}\right)
\end{aligned}
$$

In the above equations, $m_{i}$ is the transverse magnification of the i-th mirror and $a_{i}$ is the ratio of between incident heights of the marginal ray. They are defined as follows:

$$
\begin{aligned}
& m_{1}=\frac{n_{0} u_{0}}{n_{1} u_{1}}=-\frac{u_{0}}{u_{1}}=0 \\
& m_{2}=\frac{n_{1} u_{1}}{n_{2} u_{2}}=-\frac{u_{1}}{u_{2}}
\end{aligned}
$$

$$
\begin{aligned}
& m_{3}=\frac{n_{2} u_{2}}{n_{3} u_{3}}=-\frac{u_{2}}{u_{3}} \\
& a_{1}=\frac{h_{2}}{h_{1}} \\
& a_{2}=\frac{h_{3}}{h_{2}}
\end{aligned}
$$

From the first order design and condition for correcting Petzval field curvature, all of the marginal ray data $\left(h_{1}, h_{2}, h_{3}, u_{0}, u_{1}, u_{2}, u_{3}\right)$ are determined, and Eq. (22) $\sim$ Eq. (28) can be evaluated. The conic constants correcting $S_{I}, S_{I I}$ and $S_{I I I}$ are given as follows:

$$
\begin{aligned}
& k_{2}=-\frac{m_{2}^{2}\left\{a_{1} a_{2} m_{3} s_{3 f}+Q\left(m_{3}^{2} s_{2 f}-s_{2 r}\right)\right\}}{\left(a_{1}-1\right)\left(m_{2}-1\right)^{3} m_{3}\left\{Q m_{2} m_{3}-a_{1}\left(a_{1}-1\right)\right\}} \\
& k_{3}=-\frac{m_{2}\left(m_{3}^{2} s_{2 f}+s_{2 r}+Q s_{1 r}\right)+\left(a_{1}-1\right)\left(m_{2}-1\right)^{3} m_{3}^{2} k_{2}}{Q m_{2}\left(m_{3}-1\right)^{3}} \\
& k_{1}=-\frac{m_{3}^{3} s_{1 f}+a_{1} a_{2} s_{1 r}+a_{1}\left(m_{2}-1\right)^{3} m_{3}^{3} k_{2}+a_{1} a_{2}\left(m_{3}-1\right)^{3} k_{3}}{m_{2}^{3} m_{3}^{3}}
\end{aligned}
$$

\section{DESIGN EXAMPLES}

\section{Design Specifications}

In Table 1, specifications for designing of a threemirror telescope system are summarized. The telescope is a satellite camera. A CCD pixel corresponds to $0.57 \mathrm{~m}$ on the ground at observation altitude of $685 \mathrm{~km}$. Diffraction limit of the system is $14.9 \mu \mathrm{m}$ at $550 \mathrm{~nm}$ in wave length.

From the specification, some of the ray data are given by Eq. (3) (6).

TABLE 1. Design Specifications of Three-Mirror Telescope.

\begin{tabular}{c|c}
\hline \hline Altitude & $685 \mathrm{~km}$ \\
\hline Swath width & $15 \mathrm{~km}$ \\
\hline Aperture diameter & $800 \mathrm{~mm}$ \\
\hline F-number & 22.263 \\
\hline Effective focal length & $17809.439 \mathrm{~mm}$ \\
\hline Field of view(FOV) & $1.25^{\circ}$ \\
\hline Diffraction limit & $14.9 \mu \mathrm{m} \mathrm{at} \lambda=0.55 \mu \mathrm{m}$ \\
\hline $\begin{array}{c}\text { Ground sampling } \\
\text { distance(GSD) }\end{array}$ & $0.57 \mathrm{~m}$ \\
\hline
\end{tabular}




$$
\begin{aligned}
& h_{1}=400 \mathrm{~mm} \\
& u_{0}=0 \\
& u_{3}=-0.022460 \\
& \overline{u_{0}}=0.010909
\end{aligned}
$$

The paraxial angle after reflecting on the tertiary mirror, $u_{3}$ has a negative sign because we want to make an intermediate image and a real exit pupil as shown in Figure 1. A folding mirror will be located near the intermediate image or at the exit pupil.

\section{Object Conjugation Type Design}

In this type of design, the folding mirror is located on the intermediate image plane(image plane of the secondary mirror). The general characteristics of this type compared with the pupil conjugation type are as follows;

- F-ratio of the primary mirror is fast.

- Mirror separations $d_{1}$ and $d_{2}$ are short, a compact design is possible.

- It has long back focal length(BFL) and large tertiary mirror. To reduce instrumental space, an extra folding mirror may be needed.

- Only a fraction of off-axial field is available. The available fraction is taken by the folding mirror.

In designing the object conjugation type system, the basic design parameters are selected as follows:

$$
\begin{aligned}
h_{2} & =80 \mathrm{~mm} \\
d_{1} & =-1100 \mathrm{~mm} \\
d_{2} & =2000 \mathrm{~mm}
\end{aligned}
$$

The central obstruction $\sigma$, paraxial angle $u_{1}$ and f-ratio of the primary mirror $F_{1}$ are given as follows:

$$
\begin{aligned}
& \sigma=0.23011 \\
& u_{1}=-0.29091 \\
& F_{1}=1.71875
\end{aligned}
$$

From Eq. (16), the condition for correcting Petzval field curvature, we can get two solutions.

\section{Solution I}

$$
\begin{aligned}
& u_{2+}=-0.174096 \\
& h_{3}=-268.192 \mathrm{~mm} \\
& B F L=-\frac{h_{3}}{u_{3}}=-11940.856 \mathrm{~mm}
\end{aligned}
$$

\footnotetext{
Solution II

$u_{2_{-}}=-0.058631$
}

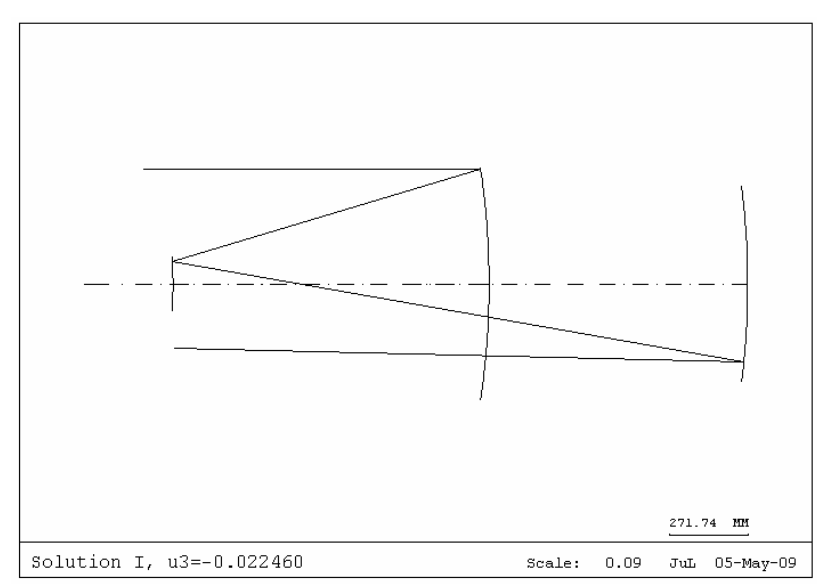

(a) Solution I

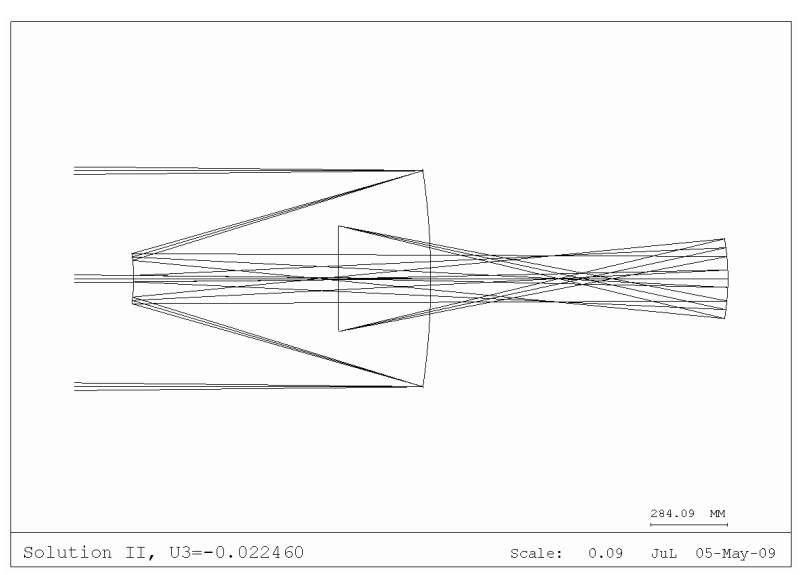

(b) Solution II

FIG. 2. Optical layout of the Solution I and II. In the Solution I, the image plane is not shown because BFL of the system is too long.

$$
\begin{aligned}
& h_{3}=-37.263 \mathrm{~mm} \\
& B F L=-\frac{h_{3}}{u_{3}}=-1659.079 \mathrm{~mm}
\end{aligned}
$$

Figure 2 shows inline optical layouts of the two solutions. The BFL of the Solution I is too long, impractical to use. In the Solution II, the tertiary mirror is quite small, but BFL is larger than the diameter of primary mirror. Hence, we need an extra folding mirror to reduce instrumental space. Figure 3 shows a compact construction of the system. Design data of the Solution II and the third order aberrations are listed in Table 2 . All of third order aberrations are well corrected except distortion. Finite ray aberrations of the Solution II are shown in Figure 3. We can see some uncorrected distortion and residual fifth order aberrations in longitudinal spherical aberration and astigmatic field curves.

\section{Pupil Conjugation Type Design}

In pupil conjugation type design, the folding mirror 


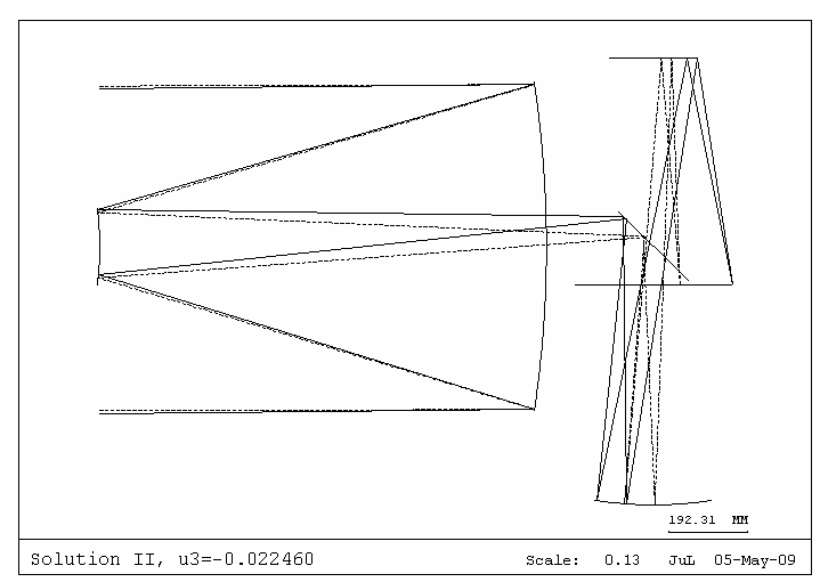

FIG. 3. Optical layout of the Solution II with two folding mirrors.

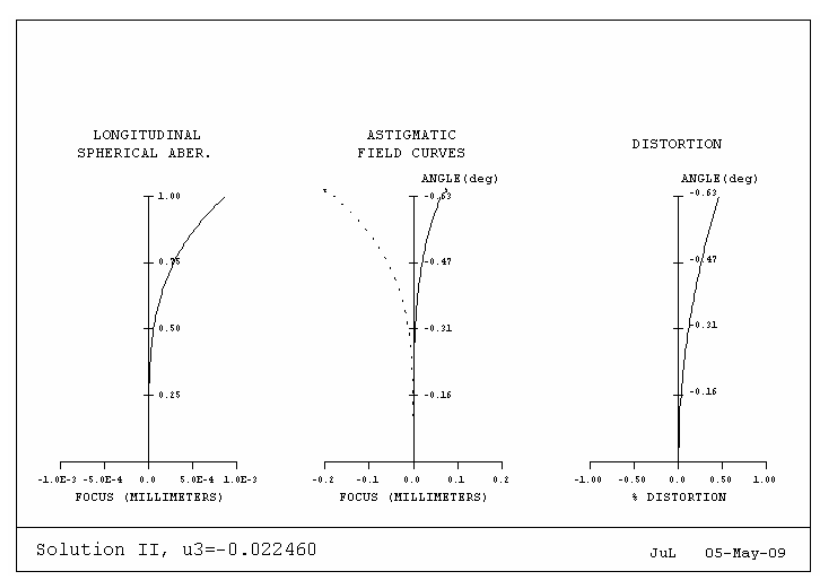

FIG. 4. Finite ray aberrations of the Solution II. which has a center hole is located at the exit pupil position. The rays incident from the secondary mirror are reflected by the folding mirror, and come to the tertiary mirror. After reflecting on the tertiary mirror, the rays pass through the center hole of the folding mirror. In this configuration, we need bigger instrumental space compared with the object conjugation type. But this type of design has a ring field and better imaging performance in off-axial field. They allow us more flexible usage of the image plane.

The following are selected design parameters.

$$
\begin{aligned}
h_{2} & =80 \mathrm{~mm} \\
d_{1} & =-1400 \mathrm{~mm} \\
d_{2} & =2800 \mathrm{~mm}
\end{aligned}
$$

Mirror separations $d_{1}, d_{2}$ are longer than those of the previous design, object conjugation type. Hence, this system has higher central obstruction, smaller paraxial angle $u_{1}$, and larger $F_{1}$.

$$
\begin{aligned}
& \sigma=0.23832 \\
& u_{1}=-0.22857 \\
& F_{1}=2.1875
\end{aligned}
$$

There are two real solutions corrected for Petzval field curvature by Eq. (16). Let's denote them Solution III and IV.

\section{Solution III}

$$
\begin{aligned}
& u_{2+}=-0.141358 \\
& h_{3}=-315.803 \mathrm{~mm}
\end{aligned}
$$

TABLE 2. Design data and third order aberrations of the Solution II.

(a) Design data

\begin{tabular}{c|c|c|c|c}
\hline$\#$ & $\mathrm{r}(\mathrm{mm})$ & $\mathrm{d}(\mathrm{mm})$ & conic constant & remark \\
\hline 1 & -2750.000 & -1100.000 & -0.985669493 & stop \\
\hline 2 & -688.831 & 2000.000 & 2.132493050 & \\
\hline 3 & -919.034 & -1659.079 & -0.638538489 & \\
\hline
\end{tabular}

(b) Third order aberrations(evaluated by Code V)

\begin{tabular}{c|c|c|c|c|c|c|c|c|c|c}
\hline & SA & TCO & TAS & SAS & PTB & DST & \multicolumn{2}{|c|}{ AX } & LAT & PTZ \\
\hline \multirow{2}{*}{$\begin{array}{c}1 \\
\text { (stop })\end{array}$} & 54.80651 & 12.33107 & 0.61653 & 0.00000 & -0.30827 & 0.00000 & 0.00000 & 0.00000 & 0.00073 \\
\cline { 2 - 10 } & -54.02110 & 0.00000 & 0.00000 & 0.00000 & & 0.00000 & \multicolumn{2}{|c|}{ ASPHERIC CONTRIBUTIONS } \\
\hline \multirow{2}{*}{2} & -12.63551 & -6.14438 & 0.234736 & 0.89870 & 1.23069 & 0.14567 & 0.00000 & 0.00000 & -0.00290 \\
\cline { 2 - 10 } & 11.89871 & -5.35425 & 0.80311 & 0.26770 & & -0.04015 & \multicolumn{2}{|c|}{ ASPHERIC CONTRIBUTIONS } \\
\hline \multirow{2}{*}{3} & 0.02200 & -0.24405 & -0.02011 & -0.62165 & -0.92242 & 2.29837 & \multicolumn{2}{|c|}{0.00000} & 0.00000 & 0.00218 \\
\cline { 2 - 10 } & -0.07061 & -0.58839 & -1.63426 & -0.54476 & & -1.51306 & \multicolumn{2}{|c|}{ ASPHERIC CONTRIBUTIONS } \\
\hline \multirow{2}{*}{ SUM } & 0.00000 & 0.00000 & 0.00000 & 0.00000 & 0.00000 & -0.89083 & \multicolumn{2}{|c|}{0.00000} & 0.00000 & 0.00000 \\
\hline
\end{tabular}




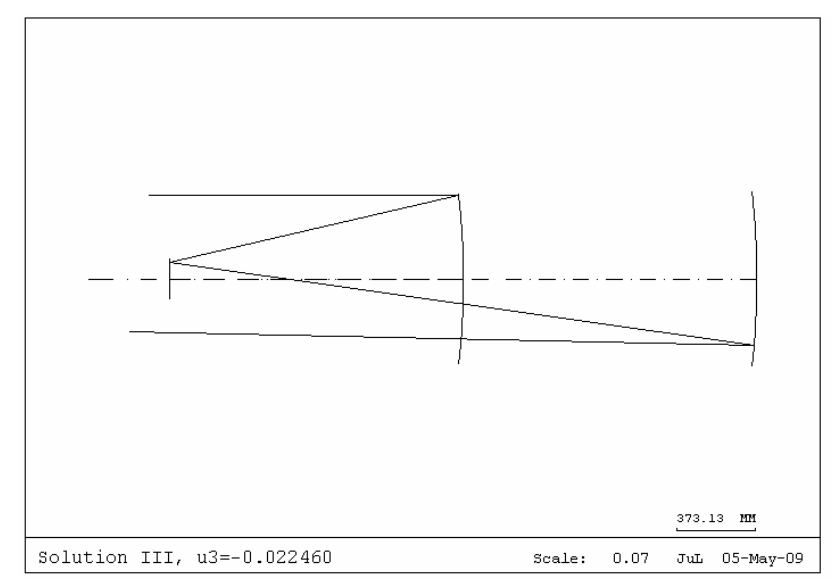

(a) Solution III

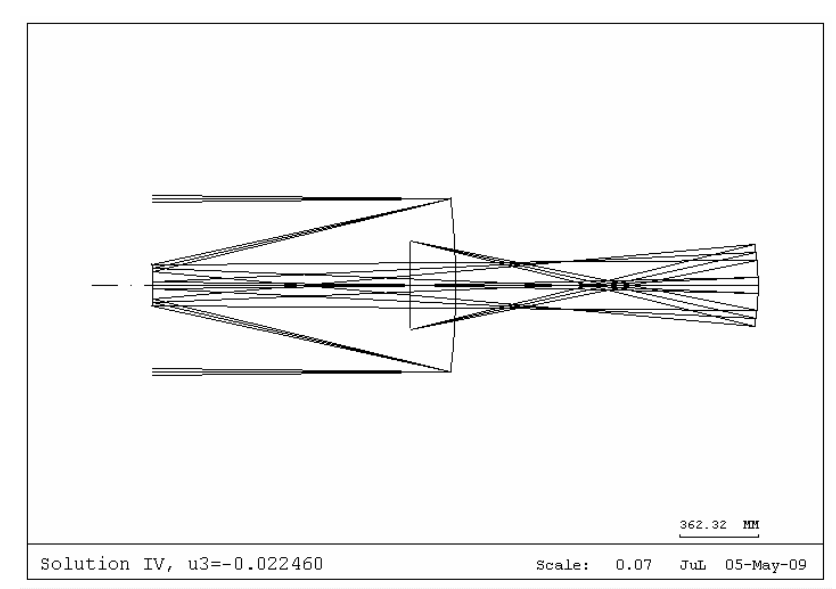

(b) Solution IV

FIG. 5. Optical layout of the Solution III and IV. In the Solution III, image plane is not shown because BFL of the system is too long.

$$
B F L=-\frac{h_{3}}{u_{3}}=-14060.699 \mathrm{~mm}
$$

Solution IV

$$
\begin{aligned}
& u_{2-}=-0.041499 \\
& h_{3}=-36.197 \mathrm{~mm} \\
& B F L=-\frac{h_{3}}{u_{3}}=-1611.608 \mathrm{~mm}
\end{aligned}
$$

Among the two solutions, the Solution III is not a reasonable one because of too long BFL. Figure 5 shows inline optical layouts of the two solutions. Figure 6 is an example of practical optical layout of the Solution IV, a folding mirror is located at the exit pupil. Design data and the third order aberrations of the Solution IV are listed in Table 3. All of the third order aberrations except distortion are also well corrected. The uncorrected distortion has slightly larger value than the Solution II, the object conjugation type. Finite ray aberrations of the Solution IV are presented in Figure 7. The astigmatic field curves show very good balancing between sagittal

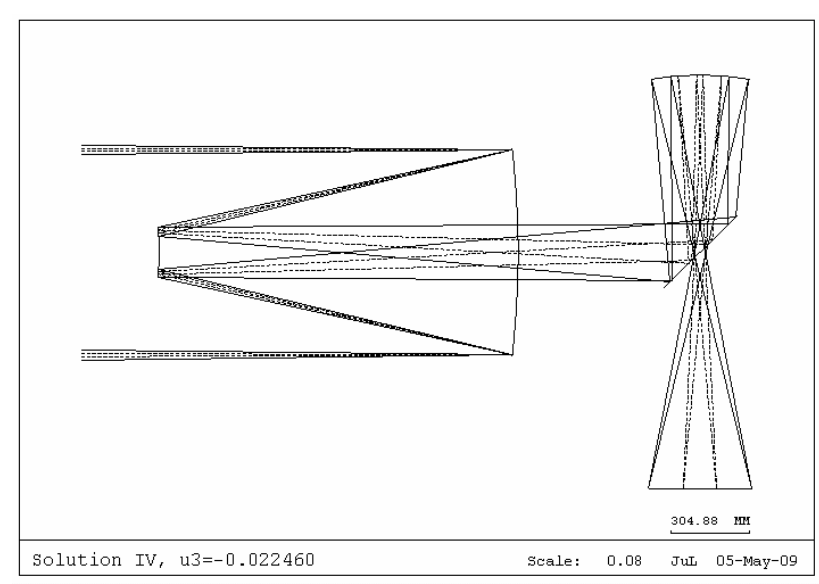

FIG. 6. Optical layout of the Solution IV with folding mirror.

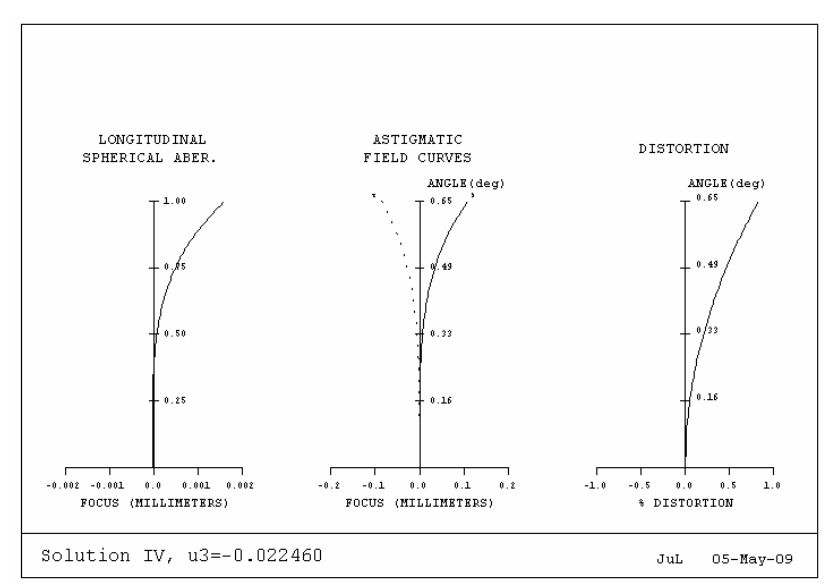

FIG. 7. Finite ray aberrations of the Solution IV.

field curvature $\mathrm{S}$ and tangential field curvature T. The residual $\mathrm{S}$ and $\mathrm{T}$ are just a half compared with those of the Solution II. Better imaging performance is expected in off-axial field.

\section{CONCLUSION}

From the viewpoint of applications, physical configuration of an optical system is important in addition to aberration correction. We present an analytic design procedure for a three-mirror system which has a suitable configuration for specific application and is corrected for four kinds of third order aberrations, spherical aberration, coma, astigmatism, and Petzval field curvature.

In designing a three-mirror system, we have eight degrees of freedom. They are three curvature radii $\left(r_{1}, r_{2}\right.$, $\left.r_{3}\right)$, three conic constants $\left(k_{1}, k_{2}, k_{3}\right)$ and two axial separations between mirrors $\left(d_{1}, d_{2}\right)$. Among the third order aberrations, spherical aberration, coma, astigmatism and distortion are linear functions of conic constants. 
TABLE 3. Design data and third order aberrations of the Solution IV.

(a) Design data

\begin{tabular}{c|c|c|c|c}
\hline$\#$ & $\mathrm{r}(\mathrm{mm})$ & $\mathrm{d}(\mathrm{mm})$ & conic constant & remark \\
\hline 1 & -3500.000 & -1400.000 & -0.982078556 & stop \\
\hline 2 & -855.283 & 2800.000 & -1.930109940 & \\
\hline 3 & -1131.875 & -1611.608 & -0.607768548 & \\
\hline
\end{tabular}

(b) Third order aberrations(evaluated by Code V)

\begin{tabular}{|c|c|c|c|c|c|c|c|c|c|}
\hline & $\mathrm{SA}$ & TCO & TAS & SAS & PTB & DST & $\mathrm{AX}$ & LAT & PTZ \\
\hline \multirow{2}{*}{$\begin{array}{c}1 \\
\text { (stop) }\end{array}$} & 26.58435 & -7.91707 & 0.52395 & 0.00000 & -0.26198 & 0.00000 & 0.00000 & 0.00000 & 0.00057 \\
\hline & -26.10792 & 0.00000 & 0.00000 & 0.00000 & & 0.00000 & \multicolumn{3}{|c|}{ ASPHERIC CONTRIBUTIONS } \\
\hline \multirow{2}{*}{2} & -6.07511 & 4.03765 & 0.17756 & 0.77389 & & -0.17145 & 0.00000 & 0.00000 & -0.00234 \\
\hline & 5.62604 & 3.35098 & 0.66530 & 0.22177 & & 0.04403 & \multicolumn{3}{|c|}{ ASPHERIC CONTRIBUTIONS } \\
\hline \multirow{2}{*}{3} & 0.00467 & 0.12583 & 0.31994 & -0.43341 & & -3.89231 & 0.00000 & 0.00000 & 0.00177 \\
\hline & -0.03203 & 0.40261 & -1.68675 & -0.56225 & & 2.35553 & \multicolumn{3}{|c|}{ ASPHERIC CONTRIBUTIONS } \\
\hline SUM & 0.00000 & 0.00000 & 0.00000 & 0.00000 & 0.00000 & -1.66420 & 0.00000 & 0.00000 & 0.00000 \\
\hline
\end{tabular}

Hence, we can correct three of them by using the conic constants. The system should have the specified focal length and if we correct one more third order aberration, Petzval field curvature, there are three remaining degrees of freedom. They could be used for selecting a suitable configuration for the system.

We take $\left(h_{2}, d_{1}, d_{2}\right)$ as the basic design parameters to get a suitable configuration for real applications, where $h_{2}$ is the incident height of marginal ray on the secondary mirror and $\left(d_{1}, d_{2}\right)$ are axial separations between mirrors. We assume that effective focal length and f-number of the system are specified in the requirements for design. The condition for correcting Petzval field curvature is expressed as a quadratic function of $u_{2}$, paraxial angle of the secondary mirror after reflection. There can be two solutions. But, if one of the solutions has a suitable configuration, the other may have an improper configuration, too long BFL mostly.

Two design examples are presented. One has a compact configuration with off-axial field. In this design, a folding mirror selecting field is located in the intermediate image plane of the secondary mirror. The other has a relatively long configuration with annular ring field. In the latter case, a folding mirror is located at the exit pupil, where it acts as a glare stop. The third order aberrations of the two designs are well corrected except for distortion. But, the latter design has good balance between the residual aberrations. It shows better off-axial imaging performance compared with the former design.

\section{ACKNOWLEDGMENT}

The authors thank Dr. Yoon Woo Lee and Dr. Ho Sun Yang for thankful discussions. This research was supported by the Korea Research Institute of Science and Standards.

\section{REFERENCES}

1. G. I. Lebedeva and A. A. Garbul, "Prospective aerospace reflective objectives,” J. Opt. Technol. 61, 610-614 (1994).

2. J. H. Lee, Y. M. Kim, E.-S. Lee, S. K. Kim, E.-E. Kim, Y. W. Choi, and D. C. Park, "An optical design of a high resolution earth observation camera for small satellites," Hankook Kwanghak Hoeji(in Korean) 8, 6-12 (2000).

3. J. H. Lee, T. S. Jang, H.-S. Yang, and S.-W. Rhee, "Optical design of a compact imaging spectrometer for STSAT3,” J. Opt. Soc. Korea 12, 262-268 (2008).

4. D. Korsch, "Closed form solution for three-mirror telescopes, corrected for spherical aberration, coma, astigmatism, and field curvature," Appl. Opt. 11, 2986-2987 (1972).

5. P. N. Robb, "Three-mirror telescopes : design and optimization," Appl. Opt. 17, 2677-2685 (1978).

6. S. K. Oh, J. U. Lee, W. G. Kwon, and K. H. Hong, "Three-spherical-mirror system corrected for three kinds of third order aberrations," Hankook Kwanghak Hoeji(in Korean) 6, 93-100 (1995).

7. J. U. Lee, K. H. Hong, and W. G. Kwon, "Optical design of three-mirror telescope system for infra-red and visible imaging," Hankook Kwanghak Hoeji(in Korean) 7, 183190 (1996).

8. S. C. Choi, H. K. Kim, and Y. S. Kim, "Optical design of flat field anstigmatic three-mirror telescope," Hankook Kwanghak Hoeji(in Korean) 8, 175-182 (1997). 
9. D. Gallagher, J. Bergstrom, J. Day, B. Martin, T. Reed, P. Spuhler, S. Streetman, and M. Tommeraasen, "Overview of the optical design and performance of the high resolution science imaging experiment(HiRISE)," Proc. SPIE 5874, 58740K (2005).

10. J.-L. Lamard, G.-D. Catherine, D. Valentini, C. Renard, T. Tournier, and J.-M. Laherrere, "Design of the high resolution optical instrument for the pleiades HR earth observation satellites," in Proc. the 5th Int'l Conf. on Space Optics (ICSO 2004) (Toulouse, France, 2004) pp. 149-156.

11. L. M. Lampton, J, M. Sholl, H. M. Krim, R. Besuner, W. C. Akerlof, G. Aldering, R. Amanullah, P. Astier, C. Baltay, E. Barrelet, S. Basa, C. Bebek, J. Bercovitz, L. Bergstrom, G. Berstein, M. Bester, R. C. Bohlin, A. Bonissent, C. R. Bower, M. Campbell, W. C. Carithers Jr., E. D. Commins, C. Day, S. E. Deustua, R. S. DiGennaro,
A. Ealet, R. S. Ellis, W. Emmett, M. Eriksson, D. Fouchez, A. Fruchter, J.-F. Genat, G. Goldhaber, A. Goobar, D. E. Groom, H. D. Heetderks, S. E. Holland, D. Huterer, W. E. Johnston, R. W. Kadel, A. Karcher, A. G. Kim, W. F. Kolbe, R. E. Lafever, J. I. Lamoureux, O. Lefevre, M. E. Levi, D. S. Levin, E. V. Linder, S. C. Loken, R. Malina, A. Mazure, T. A. McKay, S. P. McKee, R. Miquel, N. Morgan, E. Mortsell, N. Mostek, S. Mufson, J. A. Musser, P. E. Nugent, M. H. Oluseyi, R. Pain, N. P. Palaio, D. H. Pankow, S. Perlmutter, E. Prieto, D. Rabinowitz, A. Refregier, J. Rhodes, A. N. Roe, M. S. Schubnell, G. Smadja, R. Smith, G. F. Smoot, J. A. Snyder, A. Spadafora, A. Szymkowiak, G. Tarle, K. Taylor, A. Tilquin, A. D. Tomasch, D. Vincent, H. von der Lippe, J.-P. Walder, and G. Wang, "SNAP telescope: an update," Proc. SPIE 5166, 113-123 (2004).

12. W. T. Welford, Aberrations of the Symmetrical Optical System (Academic Press, London, UK, 1974). 